\title{
Cerebral Reorganization after Hemispherectomy: A DTI Study
}

\author{
(D)A. Meoded, (DA.V. Faria, (D)A.L. Hartman, (DG.I. Jallo, (DS. Mori, (DM.V. Johnston, (DT.A.G.M. Huisman, and (D)A. Poretti \\ O- $\equiv$
}

\begin{abstract}
BACKGROUND AND PURPOSE: Hemispherectomy is a neurosurgical procedure to treat children with intractable seizures. Postsurgical improvement of cognitive and behavioral functions is observed in children after hemispherectomy suggesting plastic reorganization of the brain. Our aim was to characterize changes in DTI scalars in WM tracts of the remaining hemisphere in children after hemispherectomy, assess the associations between WM DTI scalars and age at the operation and time since the operation, and evaluate the changes in GM fractional anisotropy values in patients compared with controls.
\end{abstract}

MATERIALS AND METHODS: Patients with congenital or acquired neurologic diseases who required hemispherectomy and had highquality postsurgical DTI data available were included in this study. Atlas- and voxel-based analyses of DTI raw data of the remaining hemisphere were performed. Fractional anisotropy and mean, axial, and radial diffusivity values were calculated for WM and GM regions. A linear regression model was used for correlation between DTI scalars and age at and time since the operation.

RESULTS: Nineteen patients after hemispherectomy and 21 controls were included. In patients, a decrease in fractional anisotropy and axial diffusivity values and an increase in mean diffusivity and radial diffusivity values of WM regions were observed compared with controls ( $P<.05$, corrected for multiple comparisons). In patients with acquired pathologies, time since the operation had a significant positive correlation with white matter fractional anisotropy values. In all patients, an increase in cortical GM fractional anisotropy values was found compared with controls $(P<.05)$.

CONCLUSIONS: Changes in DTI metrics likely reflect Wallerian and/or transneuronal degeneration of the WM tracts within the remaining hemisphere. In patients with acquired pathologies, postsurgical fractional anisotropy values correlated positively with elapsed time since the operation, suggesting a higher ability to recover compared with patients with congenital pathologies leading to hemispherectomy.

ABBREVIATIONS: $A B A=$ atlas-based analysis; $A D=$ axial diffusivity; $C G C=$ cingulate bundle at the cortex; $F A=$ fractional anisotropy; $M D=$ mean diffusivity; $\mathrm{RD}=$ radial diffusivity; $\mathrm{VBA}=$ voxel-based analysis

$\mathrm{H}^{2}$ emispherectomy is a neurosurgical procedure to treat children with intractable seizures that start in childhood, arise diffusely from 1 hemisphere, and are associated with unihemispheric insults. ${ }^{1,2}$ These include congenital (eg, hemimegalen-

Received August 12, 2015; accepted after revision November 3.

From the Section of Pediatric Neuroradiology (A.M., T.A.G.M.H., A.P.), Division of Pediatric Radiology, Russell H. Morgan Department of Radiology and Radiological Sciences (A.V.F., S.M.), and Departments of Neurology (A.L.H., M.V.J.) and Neurosurgery (G.I.J.), The Johns Hopkins University School of Medicine, Baltimore, Maryland; and F.M. Kirby Research Center for Functional Brain Imaging (S.M.), Kennedy Krieger Institute (M.V.J.), Baltimore, Maryland.

Susumu Mori was funded by National Institutes of Health grants: P41EB015909 and R01 NS084957.

Please address correspondence to Andrea Poretti, MD, Section of Pediatric Neuroradiology, Division of Pediatric Radiology, Russell H. Morgan Department of Radiology and Radiological Sciences, The Johns Hopkins University School of Medicine, Zayed Building Room 4174, 1800 Orleans St, Baltimore, MD 21287; e-mail: aporett1@ jhmi.edu cephaly and prenatal stroke) or postnatally acquired (eg, Rasmussen encephalitis and traumatic brain injury) lesions. ${ }^{1,3}$

Postsurgical improvement of cognitive and behavioral functions is observed in children after hemispherectomy. ${ }^{4-8}$ Motor, cognitive, and behavioral outcomes after hemispherectomy, however, differ among children and depend on the etiology and time of onset of the underlying pathology and age of the child at neurosurgery. ${ }^{6,9-11}$ These differences suggest that posthemispherectomy plastic reorganization of brain circuitry may differ on the basis of the underlying disease.

We used DTI to investigate the WM architecture of the re-

\footnotetext{
- Indicates open access to non-subscribers at www.ajnr.org

$\equiv$ Indicates article with supplemental on-line tables.

Indicates article with supplemental on-line photos.

http://dx.doi.org/10.3174/ajnr.A4647
} 
maining cerebral hemisphere in children after hemispherectomy. We performed the following: 1) characterization of the changes in DTI metrics in WM tracts of the remaining hemisphere in children after hemispherectomy, and 2) assessment of the associations between WM DTI scalars and age at and time since neurosurgery. We hypothesized the following: 1) a secondary degeneration of WM tracts in patients compared with controls, and 2) more severe changes in DTI scalars in patients with congenital compared with postnatally acquired pathologies. Finally, we evaluated the changes in GM fractional anisotropy (FA) values in patients compared with controls.

\section{MATERIALS AND METHODS}

This retrospective study was approved by the Johns Hopkins University review board.

\section{Subjects}

The inclusion criteria for this study were the following: 1) status post full anatomic hemispherectomy with sparing of only the basal ganglia and thalamus, and 2) availability of postsurgical DTI data without artifacts. Demographic data and detailed information about the etiology of intractable seizures leading to hemispherectomy (classified as "congenital" for cortical malformations and prenatally acquired lesions and "acquired" for postnatally acquired lesions), age at neurosurgery, and time interval between the operation and the DTI study were collected by a review of the clinical charts.

Controls were selected from our neuroimaging data base by using 3 criteria: 1) normal brain anatomy, 2) absence of neurologic disorders, and 3) availability of DTI raw data. MR imaging studies of the controls were acquired for clinical indications such as facial skin lesions or evaluation of soft-tissue pathologies confined to the head and neck region.

\section{DTI Acquisition}

DTI data were acquired on two $1.5 \mathrm{~T}$ scanners (Intera; Philips Healthcare, Best, Netherlands; and Avanto; Siemens, Erlangen, Germany). On the Philips scanner, a DTI dataset was acquired with a multisection, single-shot echo-planar (sensitivity encoding factor $=2.5$ ) spin-echo sequence. Diffusion-weighting was applied along 32 directions with a b-value of $700 \mathrm{~s} / \mathrm{mm}^{2}$. Five minimally weighted images ( $b$-value $=33 \mathrm{~s} / \mathrm{mm}^{2}$ ) were also acquired. For the acquisition of the DTI data, we used the following parameters: section thickness $=2.5 \mathrm{~mm}$, FOV $=240 \times 240 \mathrm{~mm}$, and matrix size $=96 \times 96$ reconstructed/interpolated to a matrix of $256 \times 256$. The acquisition was repeated 3 times to enhance the SNR.

DTI parameters on the Siemens scanner were the following: 20 noncollinear directions and a high b-value of $1000 \mathrm{~s} / \mathrm{mm}^{2}$. An additional measurement without diffusion-weighting $(b=0$ $\mathrm{s} / \mathrm{mm}^{2}$ ) was performed. For the acquisition of the DTI data, we used the following parameters: section thickness $=2.5 \mathrm{~mm}$, FOV $=240 \times 240 \mathrm{~mm}$, and matrix size $=192 \times 192$. We used parallel imaging (integrated parallel acquisition technique) $=2$ with a generalized autocalibrating partially parallel acquisition reconstruction. The acquisition was repeated twice to enhance the SNR.

\section{DTI Postprocessing}

DTI postprocessing was performed off-line by using MRI Studio software (Jiang $\mathrm{H}$ and Mori S, Johns Hopkins University; at www. MriStudio.org). The raw diffusion-weighted images were first coregistered to one of the least diffusion-weighted images and corrected for eddy current and subject motion. We generated the following map: FA, color-coded FA, trace of diffusion, axial diffusivity $(\mathrm{AD})$ and radial diffusivity (RD), and mean B0. After skull-stripping, we subsequently normalized the images to the Montreal Neurological Institute coordinates by using B0 images for both the subject and the template. Two "half-brain templates," including only 1 cerebral hemisphere (right and left, respectively), were previously created by using the JHU-MNI template (On-line Fig 1). Subsequently, a transformation by using a dual-contrast (FA and trace of diffusion) large deformation diffeomorphic metric mapping was applied.

As the next step, atlas-based analysis (ABA) was performed by using the WM parcellation map of the "half-brain JHUMNI template" to parcellate the brain into 88 anatomic regions including both GM and WM. Because of the reciprocal nature of both linear and nonlinear transformations, the transformation results were used to warp the parcellation map to the original DTI data, thus automatically segmenting each brain into the 88 subregions. After exclusion of the extracerebral spaces by a trace of diffusion threshold at 0.0045 , FA, mean diffusivity (MD), $\mathrm{AD}$, and $\mathrm{RD}$ values were calculated for following categories of tracts: 1) projection tracts: posterior limb of the internal capsule; anterior, superior, and posterior corona radiata; 2 ) association tracts: cingulate bundle at the cortex (CGC), cingulate bundle-hippocampal part, superior longitudinal fasciculus, and superior fronto-occipital fasciculus; and 3) commissural tracts: genu, body, and splenium of the corpus callosum of the remaining hemisphere. ${ }^{12}$ In addition, DTI metrics were collected for the following GM structures: superior parietal, frontal, temporal occipital, precentral, postcentral, and cingulate gyri and the thalamus and putamen. MD values were calculated using the formula:

$$
\mathrm{MD}=\text { Trace of Diffusion } / 3
$$

After ABA, voxel-based analysis (VBA) was performed by a normalization of the subject data to the template by using the large deformation diffeomorphic metric mapping matrix. Statistical parametric mapping was used to assess DTI scalar differences between all patients and controls. VBA was performed with SPM8 (http://www.fil.ion.ucl.ac.uk/spm/software/spm8) for Matlab, Version 2011b (MathWorks, Natick, Massachusetts). After masking all images with the half-brain template and flipping all images to the left side, we smoothed the images with a 10 -mm full width at half maximum filter. The postprocessed scans of the patient group were compared with the scans of controls by using a 2-sample $t$ test, with scanner as a covariate. For the statistical analysis, an explicit WM mask (FA threshold of $>0.2$ ) created with ROI EDITOR (http://cmrm.med.jhmi.edu/cmrm/forumMRI/forum. php?id=3) was used. VBA was performed for FA, MD, AD, and RD maps. In our analyses, we used false discovery rate correction for multiple comparisons with $P<.05$ and a 30 -voxel extent threshold. Finally, after controlling for correlation for right and 
Table 1: Demographic and clinical data of all patients with hemispherectomy

\begin{tabular}{|c|c|c|c|c|c|c|}
\hline Patients & Sex & $\begin{array}{c}\text { Age at Study } \\
(\mathrm{yr})\end{array}$ & $\begin{array}{c}\text { Age at Operation } \\
(y r)\end{array}$ & $\begin{array}{c}\text { Time since } \\
\text { Operation (yr) }\end{array}$ & Diagnosis & Etiology Group \\
\hline 1 & $M$ & 8.1 & 5.9 & 2.2 & HME left & Congenital \\
\hline 2 & $M$ & 3.3 & 1.1 & 2.2 & HME left & Congenital \\
\hline 3 & $M$ & 14.8 & 3.7 & 11.1 & Cortical dysplasia right $^{a}$ & Congenital \\
\hline 4 & $M$ & 2.2 & 0.9 & 1.3 & HME left & Congenital \\
\hline 5 & $\mathrm{~F}$ & 2.7 & 2.7 & 0.02 & Cortical dysplasia right $^{a}$ & Congenital \\
\hline 6 & $M$ & 12.9 & 5.7 & 7.2 & Cortical dysplasia left ${ }^{a}$ & Congenital \\
\hline 7 & $\mathrm{~F}$ & 11.5 & 6.9 & 4.6 & Cortical dysplasia right ${ }^{a}$ & Congenital \\
\hline 8 & $\mathrm{~F}$ & 0.9 & 0.8 & 0.1 & HME left & Congenital \\
\hline 9 & $M$ & 14.0 & 2.3 & 11.7 & Prenatal stroke right & Congenital \\
\hline 10 & $\mathrm{~F}$ & 8.9 & 4.1 & 4.8 & Prenatal stroke left & Congenital \\
\hline 11 & $\mathrm{~F}$ & 20.5 & 2.5 & 18.0 & Prenatal stroke right & Congenital \\
\hline 12 & $\mathrm{~F}$ & 8.7 & 2.2 & 6.5 & Rasmussen right & Acquired \\
\hline 13 & $\mathrm{~F}$ & 3.9 & 3.1 & 0.8 & Rasmussen left & Acquired \\
\hline 14 & $\mathrm{~F}$ & 18.1 & 9.8 & 8.3 & Rasmussen right & Acquired \\
\hline 15 & $\mathrm{~F}$ & 15.2 & 3.8 & 11.4 & Rasmussen right & Acquired \\
\hline 16 & $\mathrm{~F}$ & 12.2 & 4.1 & 8.1 & Rasmussen right & Acquired \\
\hline 17 & $\mathrm{~F}$ & 25.0 & 12.9 & 12.1 & Rasmussen right & Acquired \\
\hline 18 & $\mathrm{~F}$ & 20.7 & 4.4 & 16.3 & Postnatal stroke right & Acquired \\
\hline 19 & $\mathrm{~F}$ & 14.5 & 5.7 & 8.8 & Postnatal stroke right & Acquired \\
\hline
\end{tabular}

Note:- HME indicates hemimegalencephaly.

${ }^{a}$ Includes an extensive unilateral malformation of cortical development affecting $>1$ cerebral lobe requiring full anatomic hemispherectomy rather than a more conservative neurosurgical approach.

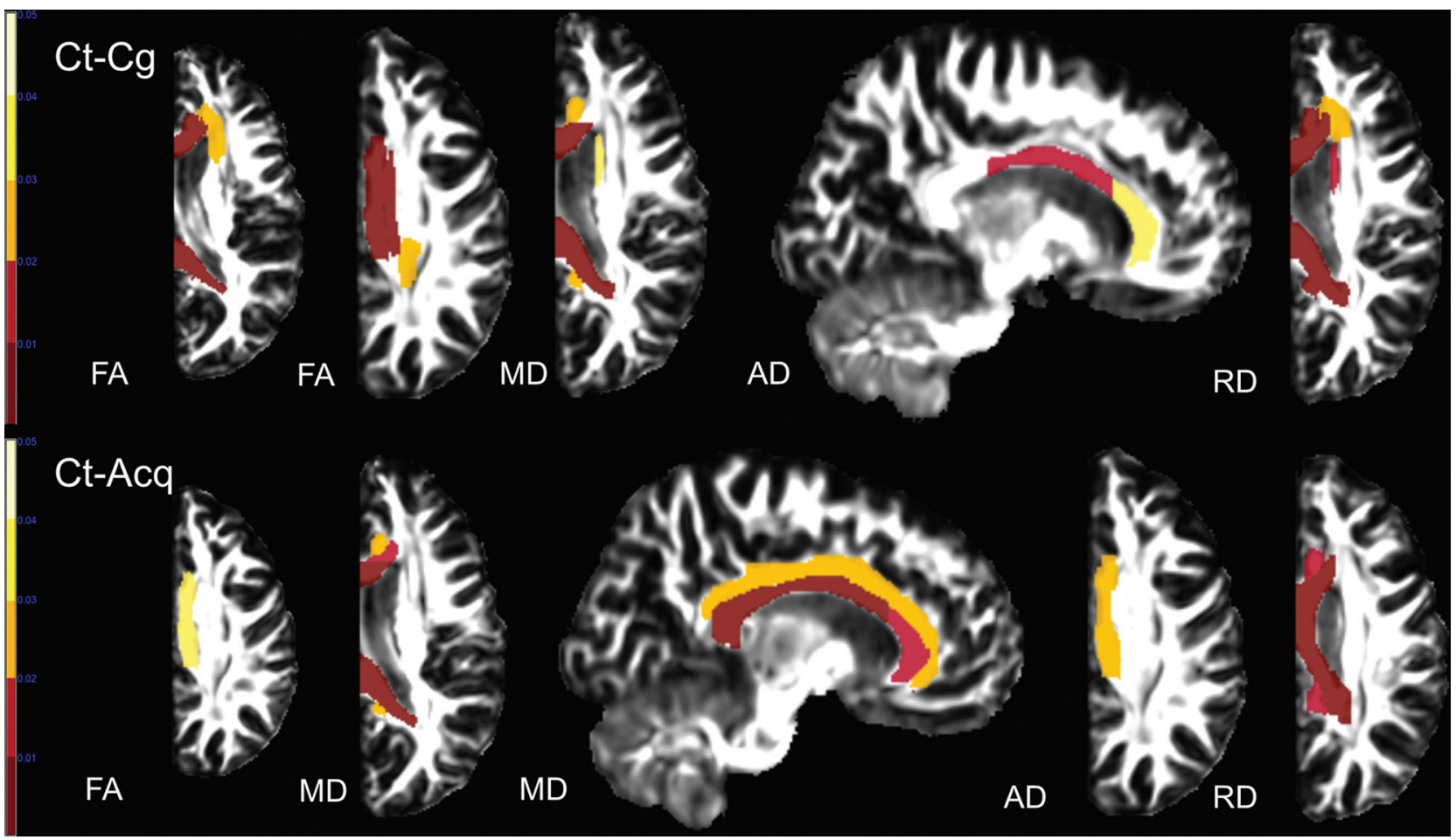

FIG 1. Results of ABA. Compared with controls (Ct), patients with anatomic hemispherectomy because of a congenital ( $\mathrm{Cg}$ ) and acquired (Acq) underlying etiology showed a decrease in FA and $A D$ and an increase in $M D$ and RD in multiple WM tracts. Only results that survived the Tukey significant difference test are depicted with $P<.05$. Color bars represent $P=.001-.05$ with a color gradient from red to light yellow. Ct-Cg indicates differences between controls and patients with congenital etiology leading to hemispherectomy; Ct-Acq indicates differences between controls and patients with acquired etiology leading to hemispherectomy.

left structures in our controls, we flipped the right half-brain to the left for all patients and controls, so analysis was done on the left hemisphere.

\section{Statistical Analysis}

Statistical analyses were performed by using SPSS, Version 21 (IBM, Armonk, New York). Data obtained from ABA analysis was compared by using a 1-way ANCOVA with scanner type as a covariate. Post hoc analyses were performed by using the Tukey significant difference test. To evaluate the associations between DTI scalars of the selected WM tracts and GM structures and age at the operation and time since the operation, we used a linear regression model in which age, sex, and disease duration were considered as covariates. In all our analyses, observed differences were considered statistically significant with $P<.05$. 


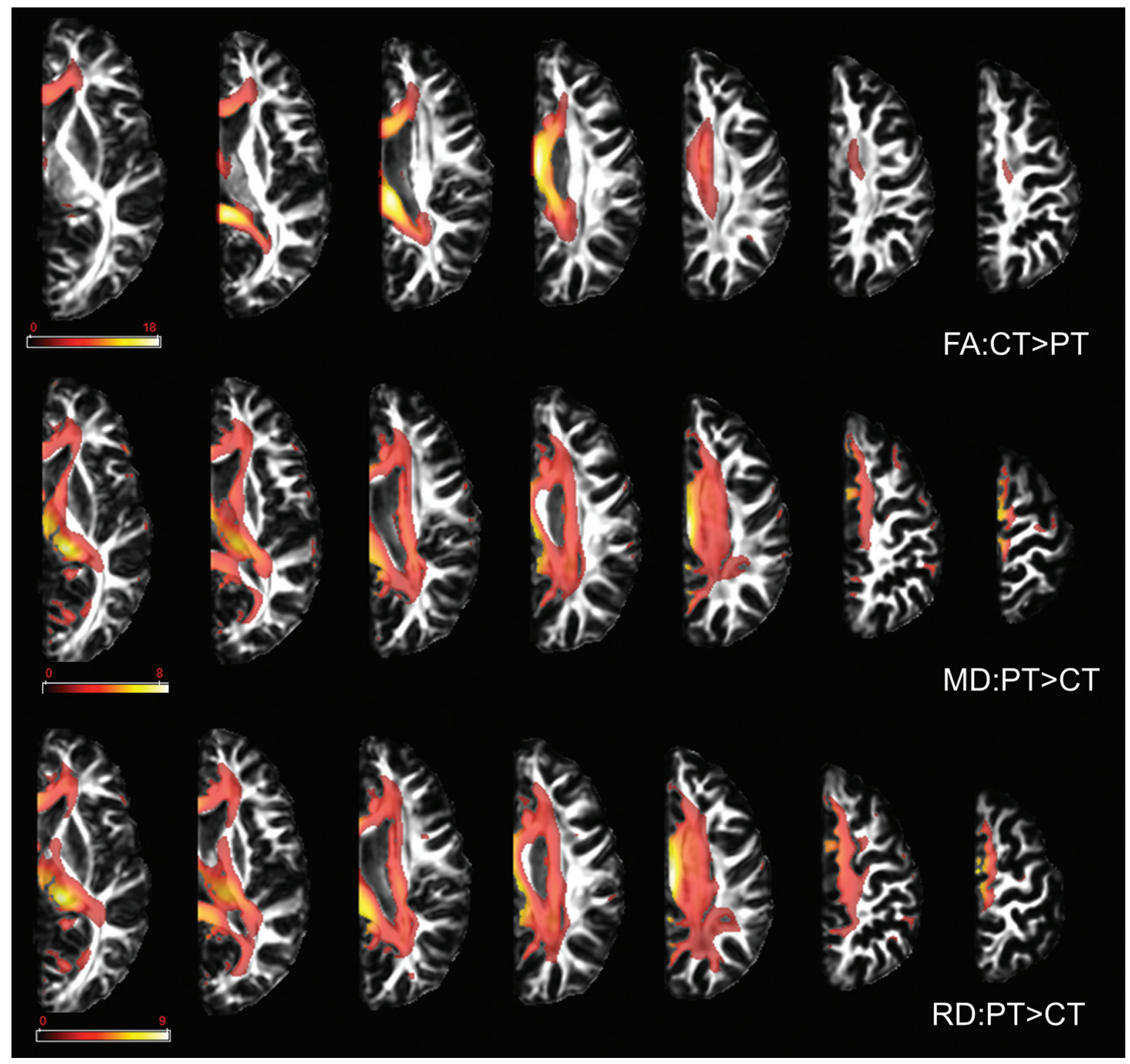

FIG 2. Results of the VBA. Compared with controls, patients showed a decrease in FA values and an increase in MD and RD values in all examined WM tracts. Only results that survived false discovery rate correction for multiple comparisons are depicted, with $P<.05$. Color bars represent the $t$ statistics. PT indicates patients; CT indicates controls.

\section{RESULTS}

\section{Subjects}

There were 19 patients (13 females; median age at MR imaging, 12.2 years; range, $0.9-25.0$ years) and 21 controls ( 14 females; median age at MR imaging, 12.2 years; range, $0.9-29.0$ years). Median age at the operation and median time since the operation were 3.8 years (range, $0.8-12.9$ years) and 7.2 years (range, $0.02-$ 18.0 years), respectively. Eleven ( 5 females) patients had a congenital etiology leading to hemispherectomy; 8 patients (all females) had an acquired etiology (Table 1). The median age at MR imaging for the patients with a congenital etiology was 8.9 years (range, 0.9-20.5 years); median age at the operation, 2.7 years (range, 0.8-6.9 years); and median time since the operation, 4.6 years (range, $0.02-18.0$ years). The median age at MR imaging for the patients with an acquired etiology was 14.9 years (range, 3.925.0 years); median age at the operation, 4.3 years (range, 2.2-12.9 years); and median time since the operation, 8.6 years (range, $0.8-16.3$ years).

\section{Atlas-Based Analysis of WM Tracts}

One-way ANOVA and post hoc analyses revealed significant changes in DTI scalars within the projection (anterior corona radiata and posterior corona radiata), association (CGC and superior fronto-occipital fasciculus), and commissural (genu of the corpus callosum, body of the corpus callosum, and splenium of the corpus callosum) tracts between patients after hemispherectomy and controls (On-line Tables 1 and 2). When significantly different, FA and $\mathrm{AD}$ values were lower, and $\mathrm{MD}$ and $\mathrm{RD}$ values, higher in patients after hemispherectomy compared with controls (Fig 1). DTI changes between both groups of patients and controls involved commissural and association tracts more than projection tracts. A higher number of differences in DTI scalars and WM 
Table 2: Significant correlations between DTI scalars in WM structures and clinical parameters in patients after hemispherectomy classified on the basis of the underlying etiology ${ }^{a}$

\begin{tabular}{|c|c|c|c|c|c|c|}
\hline $\begin{array}{c}\text { Clinical Parameter/Etiology } \\
\text { Group }\end{array}$ & DTI Scalars & WM Tracts & $\begin{array}{l}\text { Pearson Correlation } \\
\text { Coefficient }\end{array}$ & $P$ Value & $R^{2}$ & Adjusted $R^{2}$ \\
\hline \multicolumn{7}{|l|}{ Age at the operation } \\
\hline Congenital & FA & CGC & 0.840 & .001 & 0.71 & 0.67 \\
\hline \multirow[t]{4}{*}{ Acquired } & FA & PLIC & -0.737 & .037 & 0.54 & 0.47 \\
\hline & $\mathrm{RD}$ & SCR & 0.919 & .001 & 0.84 & 0.82 \\
\hline & MD & PCR & 0.884 & .004 & 0.78 & 0.75 \\
\hline & $\mathrm{RD}$ & PCR & 0.744 & .034 & 0.55 & 0.48 \\
\hline \multicolumn{7}{|l|}{ Time since the operation } \\
\hline Congenital & FA & CGC & -0.632 & .037 & 0.40 & 0.33 \\
\hline \multirow[t]{7}{*}{ Acquired } & FA & PLIC & 0.805 & .016 & 0.65 & 0.59 \\
\hline & $\mathrm{RD}$ & PLIC & -0.792 & .019 & 0.63 & 0.56 \\
\hline & $\mathrm{RD}$ & ACR & -0.802 & .017 & 0.64 & 0.58 \\
\hline & $\mathrm{RD}$ & SCR & -0.906 & .002 & 0.82 & 0.79 \\
\hline & $M D$ & PCR & -0.788 & .020 & 0.62 & 0.56 \\
\hline & $M D$ & CGC & -0.728 & .041 & 0.53 & 0.45 \\
\hline & FA & GCC & 0.722 & .043 & 0.52 & 0.44 \\
\hline
\end{tabular}

Note:-ACR indicates anterior corona radiata; GCC, genu of the corpus callosum; PCR, posterior corona radiata; SCR, superior corona radiata; PLIC, posterior limb of internal capsule.

a Pearson correlation coefficient, $r^{2}$, adjusted $r^{2}$, and $P$ value from multivariate linear regression analysis are shown.

tracts were found between patients with congenital pathologies and controls compared with patients with acquired pathologies and controls. Between patients with congenital and acquired pathologies, only FA values in the CGC were significantly higher in the acquired group (On-line Fig 2).

\section{Voxel-Based Analysis of WM Tracts}

VBA showed a decrease in FA and an increase in MD and RD values in patients after hemispherectomy compared with controls (Fig 2). Changes in FA values were similar between VBA and ABA, while changes in $\mathrm{MD}$ and $\mathrm{RD}$ were more prominent for $\mathrm{VBA}$ compared with ABA. Compared with ABA, VBA did not reveal significant changes in AD. Changes in DTI scalars involved commissural and association tracts more than projection tracts, confirming the ABA results.

\section{Correlation between WM Tract DTI Scalars and Clinical Parameters}

In patients with acquired pathologies, age at the operation showed a significant negative correlation with FA values and a significant positive correlation with $\mathrm{MD}$ and $\mathrm{RD}$ values in projection tracts (Table 2). In the same group of patients, time since the operation had a significant positive correlation with FA values and a significant negative correlation with $\mathrm{MD}$ and $\mathrm{RD}$ values in projection fibers, the cingulate bundle at the cortex, and the genu of the corpus callosum. In patients with congenital etiology, however, only FA values within the cingulate bundle at the cortex showed a significant positive correlation with age at the operation and a significant negative correlation with time since the operation.

\section{Atlas-Based Analysis and Correlation Analysis of GM Regions}

ABA of cortical GM regions revealed a significant reduction in FA values within the superior frontal, temporal, parietal, occipital, pre- and postcentral, and cingulate gyrus in all patients compared with controls (Fig 3). Within the putamen, however, FA values were significantly higher in the congenital group compared with both the acquired group and controls. $\mathrm{MD}, \mathrm{AD}$, and $\mathrm{RD}$ did not show significant differences between patients and controls; statistically significant correlations with clinical measures were not observed.

\section{DISCUSSION}

The ability of the central nervous system to adapt and reorganize following injury early in life is remarkable but has been difficult to study systematically in human infants. Data have been mostly accumulated from case studies and suggest that lesions sustained in early childhood are associated with better recovery. ${ }^{13}$ This finding indicates a prominent structural reorganization of the human brain. ${ }^{14-16}$ The posthemispherectomy brain offers a unique opportunity for studying the structural and functional neuroanatomic reorganization that underlies plasticity. Previous studies in patients after hemispherectomy applied DTI to study the reorganization of the motor and sensory tracts..$^{9,17,18}$ These studies showed marked trophic and microstructural changes within the ipsilesional corticospinal tract, while no differences were seen between the ipsi- and contralesional medial lemnisci.

We studied the contralateral (remaining) cerebral hemisphere by using ABA and VBA. ${ }^{19}$ These postprocessing approaches are considered complementary. ${ }^{20} \mathrm{ABA}$ groups voxel values within a segmentation, resulting in a marked reduction of information (from $>1$ million voxels within a brain to 176 anatomic regions). This reduction (voxel averaging) may lead to a higher statistical power, reduction of noise, and an easier biologic interpretation. VBA, however, has a higher sensitivity to detect subtle abnormalities that may remain undetected by ABA. For both ABA and VBA, we used a half-brain template to avoid inaccurate normalization due to ipsilateral brain tissue remnants after anatomic hemispherectomy (eg, the thalamus). The accuracy of the brain parcellation hinges on the accuracy of the image transformation and subsequent atlas warping. Previous studies have shown a high level of accuracy by using the large deformation diffeomorphic metric mapping algorithm for populations with marked anatomic distortions, various disease models, and different image conditions. $^{21,22}$ In our opinion, the use of a half-brain template and 


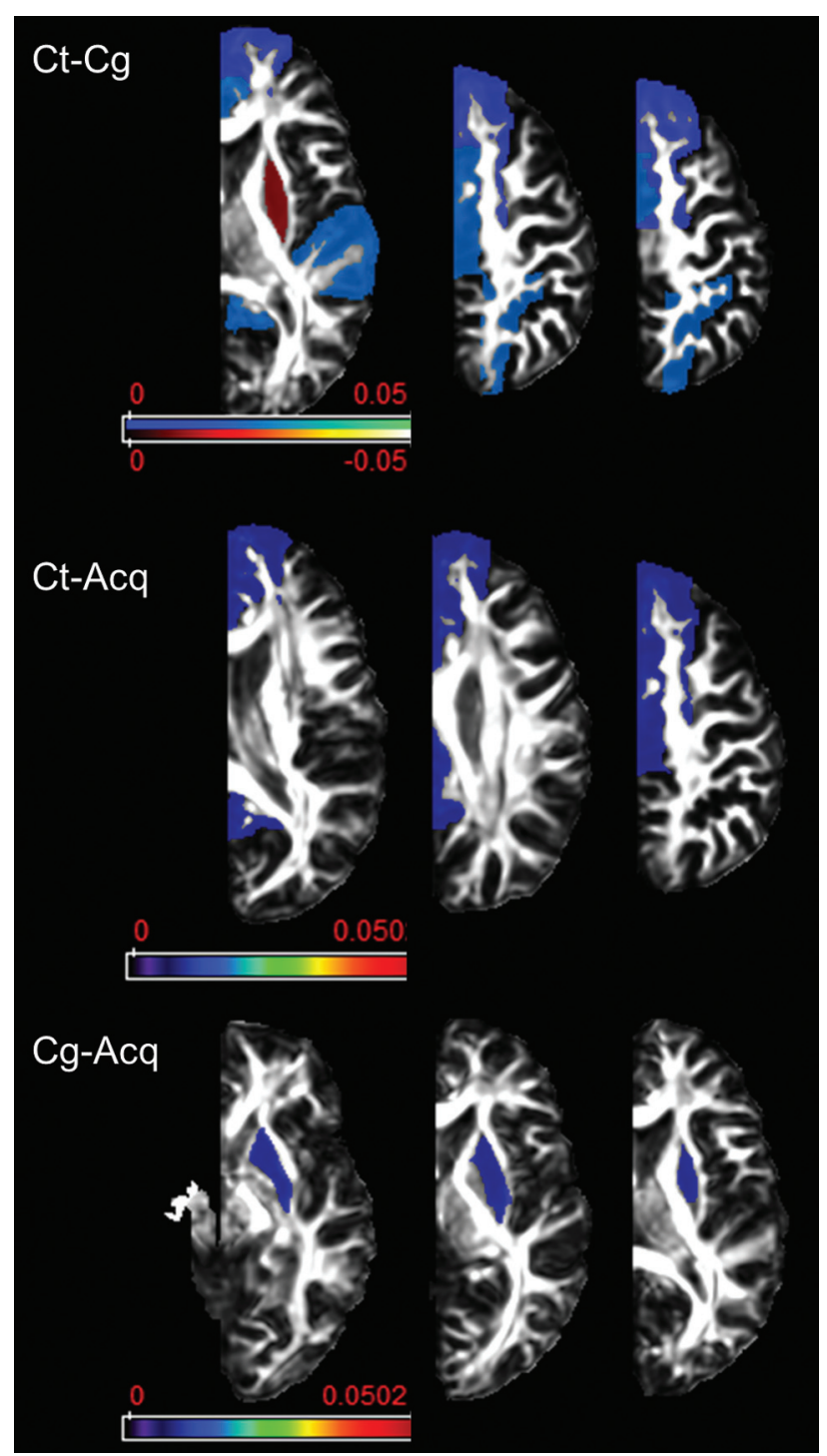

FIG 3. Results of atlas-based analysis of GM structures. Compared with controls, patients in both etiology groups showed decreased FA in all the examined cortical areas with the exception of the putamen, which showed increased FA in the congenital group. Only results that survived the Tukey significant difference test are depicted, with $P<$ .05. The Color bar represents the $P$ value: blue-light blue represents PT $<\mathrm{CT}$, and red-light yellow represents $\mathrm{CT}<\mathrm{PT}$. PT indicates patients including $\mathrm{Cg}$ (congenital) and Acq (acquired) pathologies; CT, controls..

ABA as a low granularity filter helped to improve the parcellation accuracy in this study. Although state-of-the-art methods were used in all the steps, we cannot completely exclude minor inaccuracies within brain mapping.

In our study, ABA and VBA provided similar results, including a decrease in FA values and an increase in MD and RD values in the cerebral WM tracts of patients after hemispherectomy compared with controls. In addition, ABA revealed reduced $A D$ values in cerebral WM tracts of patients compared with controls. Reduction in FA and $\mathrm{AD}$ associated with an increase in MD and RD is suggestive of secondary Wallerian and/or transneuronal degeneration. ${ }^{23}$ These changes in DTI scalars may indicate an increase in isotropic tissue diffusion characteristics consistent with the presence of gliosis and the possible increase in extracellular ma- trix as shown by histology in Wallerian and/or transneuronal degeneration.

Between patients with congenital and acquired pathologies, the only significant difference was found in FA values of the CGC, which were lower in the congenital group. A previous fMRI study showed activation in the cingulate cortex after passive foot dorsiflexion in patients after hemispherectomy. ${ }^{24}$ This may suggest a role of the CGC in rewiring cortical regions after hemispherectomy and assuming new functions such as motor tasks. Higher FA values suggest a better reorganization in the acquired group, matching the better motor recovery in these patients. ${ }^{10}$

Highly different correlations were seen between both patient groups and age at the operation and time since the operation. In the acquired group, age at the operation negatively correlated with FA values but had a positive correlation with $\mathrm{MD}$ and $\mathrm{RD}$ values in various components of the corona radiata. This finding suggests a more severe degeneration of projection fibers in children who underwent the operation at an older age. In this group, an early operation may save the contralesional WM tracts from progressive degeneration and may support/promote the greatest postsurgical improvement with the application of rehabilitation techniques earlier during brain development. ${ }^{25}$ Time since the operation, however, correlated well with an increase (normalization) of FA values and reduction of $\mathrm{MD}$ and $\mathrm{RD}$ values in WM tracts. This correlation suggests that patients with an acquired pathology have a progressive neuronal reorganization.

For the congenital group, we found less obvious results. Our structural findings match previous outcome studies that show poorer posthemispherectomy outcome in patients with congenital etiologies. ${ }^{5,10}$ In patients with congenital pathologies, structural and functional abnormalities have been shown within the "healthy" cerebral hemisphere. ${ }^{26,27}$ Our findings are contrary to the Kennard principle suggesting that the immature brain should be able to recover more easily from injury than the more developed brain. ${ }^{28}$ More recent studies have shown that lesions occurring during critical phases of brain development may result in severe disabilities caused by disturbed development of the neuronal networks.

In cortical GM regions, $\mathrm{ABA}$ revealed a decrease in FA values in patients compared with controls. The reduction in anisotropy may reflect increased unoccupied intracellular space due to a change in neuronal cell attenuation and/or reduction of the cortical neuropil. However, the significance of FA in GM is still poorly understood. Compared with WM, various cell types (eg, neuronal cell bodies, randomly oriented axons, dendritic fibers, oligodendrocytes) and extracellular matrices are present in GM structures and may affect the diffusion properties and degree of anisotropy. ${ }^{29,30}$ Putaminal FA values were significantly higher in the congenital group compared with both the acquired group and controls. This is an unexpected finding. Recently, increase in FA in GM structures after mild traumatic brain injury was shown to reflect progressive gliosis. ${ }^{31}$ In this study, it is unclear whether an increase in putaminal FA may reflect pre- or postsurgical gliosis in patients with congenital etiologies.

We are aware of some limitations of our study. Prehemispherectomy DTI data were not available for this study. Accordingly, we cannot exclude the possibility that presurgical WM mi- 
crostructural alterations may partially explain postsurgical DTI changes. The number of patients was rather small. However, few previous pediatric studies are available due to the rarity of the surgical procedure. The 2 groups of patients are of different median ages and have different age distributions. With progressing brain maturation, DTI scalars evolve. However, the most important age-related changes in DTI scalars occur within the first 4 years of life. ${ }^{32-34}$ Both studied groups of patients were markedly older (median ages of 8.9 and 14.9 years, respectively). The retrospective nature of this study made it impossible to correlate longitudinally collected clinical parameters of motor and cognitive functions and DTI scalars. Future prospective studies should include presurgical DTI data and longitudinally collected clinical parameters of neurologic functions to evaluate neuroplasticity in children after anatomic hemispherectomy more accurately.

\section{CONCLUSIONS}

DTI is an ideal tool to study brain reorganization and may shed light on the structural cerebral plasticity in patients after hemispherectomy. Changes in DTI metrics reflect Wallerian and/or transneuronal degeneration of the commissural, association, and projection WM tracts within the remaining hemisphere. In patients with acquired pathologies, postsurgical FA values tend to normalize with time, suggesting a higher potential for recovery compared with patients with congenital etiologies leading to hemispherectomy.

\section{ACKNOWLEDGMENTS}

We are thankful to Amy Bastian, $\mathrm{PhD}$, Kennedy Krieger Institute, Baltimore, Maryland, for sharing imaging data of some patients with us.

Disclosures: Andreia V. Faria—RELATED: Grant: National Institutes of Health National Institute of Biomedical Imaging and Bioengineering (R03 EB014357).* Adam L. Hartman—UNRELATED: Board Membership: Wiley Publishers (Associate Editor for Epilepsia); Expert Testimony: medicolegal work; Grants/Grants Pending: National Institute of Neurological Disorders and Stroke, * TEDCO, ${ }^{*}$ Johns Hopkins University, ${ }^{*}$ Harrington Foundation,* Howard Hughes Medical Institute*; Patents (planned, pending, or issued): Patent Cooperation Treaty filed (to be prosecuted in 2016); Royalties: Wiley, Wolters/Kluwer, Taylor \& Francis; Travel/Accommodations/ Meeting Expenses Unrelated to Activities Listed: American Academy of Pediatrics, American Epilepsy Society. George I. Jallo_UNRELATED: Expert Testimony: several defense malpractice cases; Royalties: Plural Publishing Inc, Thieme, Springer. Susumi Mori-UNRELATED: Consultancy: AnatomyWorks; Grants/Grants Pending: National Institutes of Health grants*; Patents (planned, pending, or issued): patents, One issued patent about 3D tractograph reconstruction (this technology uses the results of DTI and 3D reconstructs white matter pathway; this technology was not used in this manuscript) and 12 invention disclosures related to postprocessing of MR images including quantitative analysis and feature extraction of MR images (these disclosures are related to quantitative analysis of MR images and extraction, storing, and comparison of anatomical features in the images; these disclosures include some approaches of structural labeling that have been used in this article); Royalties: Academic Press, Elsevier; Travel/Accommodations/Meeting Expenses Unrelated to Activities Listed: business travel paid by AnatomyWorks; OTHER RELATIONSHIPS: Cofounder of AnatomyWorks. *Money paid to the institution.

\section{REFERENCES}

1. Vining EP, Freeman JM, Pillas DJ, et al. Why would you remove half a brain? The outcome of 58 children after hemispherectomy-the Johns Hopkins experience: 1968 to 1996. Pediatrics 1997;100:163-71 CrossRef Medline

2. Griessenauer CJ, Salam S, Hendrix P, et al. Hemispherectomy for treatment of refractory epilepsy in the pediatric age group: a systematic review. J Neurosurg Pediatr 2015;15:34-44 CrossRef Medline

3. Kossoff EH, Vining EP, Pillas DJ, et al. Hemispherectomy for intractable unihemispheric epilepsy etiology vs outcome. Neurology 2003; 61:887-90 CrossRef Medline

4. Kossoff EH, Vining EP, Pyzik PL, et al. The postoperative course and management of $\mathbf{1 0 6}$ hemidecortications. Pediatr Neurosurg 2002;37: 298-303 CrossRef Medline

5. Jonas R, Nguyen S, Hu B, et al. Cerebral hemispherectomy: hospital course, seizure, developmental, language, and motor outcomes. Neurology 2004;62:1712-21 CrossRef Medline

6. Pulsifer MB, Brandt J, Salorio CF, et al. The cognitive outcome of hemispherectomy in 71 children. Epilepsia 2004;45:243-54 CrossRef Medline

7. Battaglia D, Chieffo D, Lettori D, et al. Cognitive assessment in epilepsy surgery of children. Childs Nerv Syst 2006;22:744-59 CrossRef Medline

8. Devlin AM, Cross JH, Harkness W, et al. Clinical outcomes of hemispherectomy for epilepsy in childhood and adolescence. Brain 2003; 126:556-66 CrossRef Medline

9. Wakamoto H, Eluvathingal TJ, Makki M, et al. Diffusion tensor imaging of the corticospinal tract following cerebral hemispherectomy. J Child Neurol 2006;21:566-71 CrossRef Medline

10. van der Kolk NM, Boshuisen K, van Empelen R, et al. Etiology-specific differences in motor function after hemispherectomy. Epilepsy Res 2013;103:221-30 CrossRef Medline

11. Zhang J, Mei S, Liu Q, et al. fMRI and DTI assessment of patients undergoing radical epilepsy surgery. Epilepsy Res 2013;104:253-63 CrossRef Medline

12. Wakana S, Jiang H, Nagae-Poetscher LM, et al. Fiber tract-based atlas of human white matter anatomy. Radiology 2004;230:77-87 CrossRef Medline

13. Werth R. Visual functions without the occipital lobe or after cerebral hemispherectomy in infancy. Eur J Neurosci 2006;24:2932-44 CrossRef Medline

14. Bittar RG, Ptito A, Reutens DC. Somatosensory representation in patients who have undergone hemispherectomy: a functional magnetic resonance imaging study. J Neurosurg 2000;92:45-51 CrossRef Medline

15. Holloway V, Gadian DG, Vargha-Khadem F, et al. The reorganization of sensorimotor function in children after hemispherectomy: a functional MRI and somatosensory evoked potential study. Brain 2000;123(pt 12):2432-44 Medline

16. Olausson $\mathrm{H}, \mathrm{Ha} \mathrm{B}$, Duncan $\mathrm{GH}$, et al. Cortical activation by tactile and painful stimuli in hemispherectomized patients. Brain 2001; 124:916-27 CrossRef Medline

17. Mori H, Aoki S, Abe O, et al. Diffusion property following functional hemispherectomy in hemimegalencephaly. Acta Radiol 2004; 45:778-81 CrossRef Medline

18. Choi JT, Vining EP, Mori S, et al. Sensorimotor function and sensorimotor tracts after hemispherectomy. Neuropsychologia 2010;48: 1192-99 CrossRef Medline

19. Oishi K, Faria AV, Yoshida S, et al. Quantitative evaluation of brain development using anatomical MRI and diffusion tensor imaging. Int J Dev Neurosci 2013;31:512-24 CrossRef Medline

20. Faria AV, Zhang J, Oishi K, et al. Atlas-based analysis of neurodevelopment from infancy to adulthood using diffusion tensor imaging and applications for automated abnormality detection. Neuroimage 2010;52:415-28 CrossRef Medline

21. Oishi K, Faria A, Jiang H, et al. Atlas-based whole brain white matter analysis using large deformation diffeomorphic metric mapping: application to normal elderly and Alzheimer's disease participants. Neuroimage 2009;46:486-99 CrossRef Medline

22. Djamanakova A, Faria AV, Hsu J, et al. Diffeomorphic brain mapping based on T1-weighted images: improvement of registration accuracy by multichannel mapping. J Magn Reson Imaging 2013;37: 76-84 CrossRef Medline

23. Pierpaoli C, Barnett A, Pajevic S, et al. Water diffusion changes in 
Wallerian degeneration and their dependence on white matter architecture. Neuroimage 2001;13:1174-85 CrossRef Medline

24. de Bode S, Firestine A, Mathern GW, et al. Residual motor control and cortical representations of function following hemispherectomy: effects of etiology. J Child Neurol 2005;20:64-75 CrossRef Medline

25. Fritz SL, Rivers ED, Merlo AM, et al. Intensive mobility training postcerebral hemispherectomy: early surgery shows best functional improvements. Eur J Phys Rehabil Med 2011;47:569-77 Medline

26. Jahan R, Mischel PS, Curran JG, et al. Bilateral neuropathologic changes in a child with hemimegalencephaly. Pediatr Neurol 1997; 17:344-49 CrossRef Medline

27. Salamon N, Andres M, Chute DJ, et al. Contralateral hemimicrencephaly and clinical-pathological correlations in children with hemimegalencephaly. Brain 2006;129:352-65 Medline

28. Dennis M. Margaret Kennard (1899-1975): not a 'principle' of brain plasticity but a founding mother of developmental neuropsychology. Cortex 2010;46:1043-59 CrossRef Medline

29. Komlosh ME, Horkay F, Freidlin RZ, et al. Detection of microscopic anisotropy in gray matter and in a novel tissue phantom using dou- ble pulsed gradient spin echo MR. J Magn Reson 2007;189:38-45 CrossRef Medline

30. Yoshida S, Oishi K, Faria AV, et al. Diffusion tensor imaging of normal brain development. Pediatr Radiol 2013;43:15-27 CrossRef Medline

31. Budde MD, Janes L, Gold E, et al. The contribution of gliosis to diffusion tensor anisotropy and tractography following traumatic brain injury: validation in the rat using Fourier analysis of stained tissue sections. Brain 2011;134:2248-60 CrossRef Medline

32. Hermoye L, Saint-Martin C, Cosnard G, et al. Pediatric diffusion tensor imaging: normal database and observation of the white matter maturation in early childhood. Neuroimage 2006;29:493-504 CrossRef Medline

33. Saksena S, Husain N, Malik GK, et al. Comparative evaluation of the cerebral and cerebellar white matter development in pediatric age group using quantitative diffusion tensor imaging. Cerebellum 2008;7:392-400 CrossRef Medline

34. Cancelliere A, Mangano FT, Air EL, et al. DTI values in key white matter tracts from infancy through adolescence. AJNR Am J Neuroradiol 2013;34:1443-49 CrossRef Medline 